\title{
Fragmentation of fixated line stimuli as a function of gravitational orientation
}

\author{
STEPHEN R. ELLIS \\ McGill University, Montreal, Quebec, Canada
}

\begin{abstract}
The differential propensity of fixated line stimuli to fragment and disappear from view was studied as a function of the gravitational orientation of the stimuli. The propensity to fragment was measured in terms of three intercorrelated dependent variables: the number of fragmentations per fixation period, the total duration of fragmentation per fixation period, and the latency to the first fragmentation. Unlike some anisotropic visual phenomena, which may reflect orientation-independent aspects of pattern perception, the observed anisotropy of propensity to fragment can be attributed overwhelmingly to the retinal orientation of the stimuli. Accordingly, this property of fragmentation need not be ascribed to higher order aspects of pattern perception.
\end{abstract}

Several visual phenomena vary predictably when the stimuli used to demonstrate them are presented in different visual orientations. Ogilvie and Taylor (1958), for example, reported that for human subjects the detectability of obliquely oriented fine wires was inferior to that of identical wires oriented either horizontally or vertically. Similarly, Craig and Lichtenstein (1953) reported a type of Troxler effect (Troxler, 1804), showing that fixated, high-contrast line stimuli appear to fragment and disappear from view as a function of their orientation in the visual field: vertical and horizontal stinuli fragmenting least frequently, obliques fragmenting most frequently. Taylor (1963) later pointed out that other visual functions. such as bearing estimation, dot location, and adjustment of lines to parallelism, all show similar variation: each function is most accurate with vertical or horizontal stimuli.

These phenomiena have been collectively termed the "oblique effect" by Appelle (1972). The category extends to include visual contrast sensitivity for gratings when measured either psychophysically (Campbell, Kulikowski, \& Levinson, 1966; Mitchell, Freeman, \& Westheimer, 1967) or electrophysiologically in terms of evoked potentials (Campbell \& Maftei. 1970). These latter demonstrations are particularly important since the test gratings used to measure sensitivity were produced by laser interference patterns on the observer's retina, a procedure that bypasses the dioptrics of the eye. Accordingly, the variations in visual sensitivity as a function of grating orientation can be ascribed to neural rather than optical properties of the visual system. The locus of the substrate of the variation is

The author was supported by a grant from the National Research Council of Canada (No. A7891) to Dr. Ronald Melzack during the conduct of this experiment. The author's present address is: Psychology Department, Brown University, Providence. Rhode Island 02912. probably more central than the retina, since the difference between horizontal/vertical and oblique stimuli is not evident in the electroretinogram (Maffei \& Campbell. 1970). This conclusion has been supported by Manstield's (1974) recent report of an anisotropy in the distribution of the retinal orientation of foveal receptive fields of cells in Macaque striate cortex, vertical/horizontal orientations being most frequent. This anisotropy was found to be reduced for tields in more peripheral vision. Such an anisotropy of human receptive tield orientation cou!d possibly explain many psychophysical aspects of the "oblique effect."

Attempts have been made to determine whether the retinal or gravitational orientation of the stimuli was the critical factor for observing "oblique effects." Higgins and Stutz (1948), for example, noted that the meridional variation in visual acuity, as measured by the separation threshold for parallel lines, was higher along the retinal vertical/horizontal meridia regardless of head tilt. Luria (1963) more recently tested visual acuity in scotopic vision using a checkerboard test stimulus viewed during various types of 45-deg head tilt. He reported an "oblique effect" that was strongest during head-upright testing, intermediate during combined head and body tilt yielding a total 45-deg tilt. and smallest when the head alone was tilted. This result is, however, complicated by the Fourier spectra of the checkerboard test stimuli which have strong spectral components along the oblique axes, a feature which reduces the difference in acuity between horizontal/ vertical test tields and those rotated $45 \mathrm{deg}$. Additionally, the uncontrolled and irregular countertorsional eye movements that occur during prolonged head tilt (Miller, 1962) may have contributed to Luria's result in two ways: (1) Countertorsion would reduce the magnitude of the "oblique effect" during head tilt by causing 
gravitationally vertical and 45-deg oblique test gratings not to be projected, respectively, onto the $45-\mathrm{deg}$ oblique vertical retinal meridia. (2) The irregular change in the specific amount of countertorsion and corresponding change in retinal orientation would add variability to the data collected during head tilt. Thus, in the absence of eye-position data corresponding to his experimental conditions, Luria's findings cannot be conclusive. ${ }^{1}$

Further investigation of the frame of reference in which "oblique effects" occur were ind irectly inspired by reports that the visual receptive fields of cells in cat visual cortex seemed to demonstrate orientation constancy by changing their position on the retina during head or body tilt (Denney \& Adorjani, 1972; Horn \& Hill, 1969; Horn. Stechler, \& Hill, 1972; but see Schwartzkroin, 1972). Since earlier studies by Jung. Kornhuber, and da Fonseca (1963) had anticipated such results, these reports stimulated human psychophysical investigations attempting to identify a perceptual correlate of the presumptive change in receptive field orientation: Mitchell and Blakemore (1972), Ellis (1974), Lennie (1974), and Findlay and Parker (1972). The latter two of these studies used the "oblique effect" as determined by measurements of photopic visual sensitivity. Reasoning that the observed anisotropy of human visual sensitivity retlects neural processes in the visual cortex, these investigators expected the retinal orientation of the axes of minimum and maximum sensitivity to counterrotate during head tilt. In both studies, however, the axes remained retinally locked and no evidence for retinal counterrotation of receptive field axes was found.

Retinal locking is not, however, characteristic of all forms of the "oblique effect." Attneave and Olson (1967) and Attneave and Reid (1968) examined the systematic variation in human reaction times for recalling arbitrary names associated with lines of various orientations. They found that faster reaction times were associated with perceptually vertical and horizontal orientations regardless of head position. Another type of "oblique effect" which is not retinally locked is illustrated by Gibson's (1937; Gibson \& Radner, 1937) classic observation that slightly tilted lines appear less tilted after continuous inspection. Since this so-called "normalization" does not occur for vertical/horizontal lines, it qualifies as an example of the "oblique effect"; all such effects are essentially statements regarding the special properties of horizontal/vertical stimuli vs. obliques. Gibson's classic effect could be explained in terms of an anisotropy in cell-population fatigue properties parallel to the reported anisotropy in receptive field orientation (Mansfield, 1974). However, unlike the anisotropy in visual sensitivity which may be similarly explained, "normalization" has been reported to occur with respect to the gravitational vertical
(Prentice \& Beardslee, 1950; also Coltheart \& Cooper. 1972; Day \& Wade, 1969).

In view of the nonunitary character of the "oblique effects" orientation constancy, it was decided to investigate its orientation constancy using Craig and Lichtenstein's (1953) technique for studying the propensity of fixated line stimuli to gragment. Since apparent fragmentations of relatively stabilized images have been attributed to higher order perceptual processes (Donderi \& Kane, 1965; Hebb, 1963; Prichard, Heron, \& Hebb, 1960), it was anticipated that this example of the "oblique effect" might reflect spatial perception and exhibit orientation constancy. ${ }^{2}$

\section{METHODS}

\section{Subjects}

Eight male and six female undergraduate students at McGill University served as paid, naive subjects. All subjects viewed the stimuli monocularly with the dominant eye. which for all had visual function adequate for reading and for driving a car without correction as determined by the Quebec Bureau of Motor Vehicles vision test. In addition, the subjects were screened for serious astigmatism by having them view monocularly a mesopically illuminated cart-whed pattern positioned approximately $1.3 \mathrm{~m}$ directly in front of eacy eye. The pattern was drawn on white cardboard and consisted of India ink lines 3 min wide and 5 deg long radiating outward at 10 -deg intervals from a central circle $2 \mathrm{deg}$ in diam. The prospective subjects were simply asked if they noticed that any of the radiating lines appeared darker than the others. Only those not consistently identifying a particular line as darker were used as subjects. In this regard, it should be noted that though the stimuli presented in the following experiment were in peripheral vision, their eceentricity did not exceed 6 deg. Thus, the substantial astigmatism in peripheral vision which becomes important for eccentricities exceeding $10 \mathrm{deg}$ would not substantially affect the visibility of the stimuli used (Ferree, Rand, \& Hardy, 1933; Millodot \& Lamont, 1971).

\section{Stimuli and Apparatus}

The subject viewed a length of thin wire 15 deg $\times 3$ min at the subject's eye) which radiated outward from an annulus that had an outside diameter subtending 2 deg and which was made of the same wire (see bottom of Figure 1). The annulus was made by wrapping the wire around a clear Plexiglas disk and soldering the radiating wire to the edge. The entire stimulus was backmounted on an iron rod tirmly glued in a hole in the center of the disk. Directly in front of the hole, a small black bead $(5-\mathrm{min}$ diam) was glued in the center of the disk to provide a tixation point. The entire stimulus was positioned in front of a white, hemispherical, plastic diffusing screen so that it was $1.3 \mathrm{~m}$ from the subject's eye. The rod on which the stinuulus was mounted was passed through a small hole drilled in the screen so that the stimulus orientation could be adjusted by rotating the rod. The stimulus was back-illuminated by a circular 22-W cool-white fluorescent light mounted behind the diffusing screen and encircling the projecting rod. The luminance of the screen in the vicinity of the stimulus was measured to be approximatcly $117 \mathrm{~mL}$ with a SEI spot photometer. The opaque part of the stimulus only had a brightness approaching $.01 \mathrm{~mL}$ so that the stimulus had extremely high contrast. ${ }^{3}$

Care was taken to present the stimulus so that no vertical or horizontal contours would be present in the visual field. This was important. since it has been shown that parallel lines tend to inferact with each other when stabilized in the visual field (Prichard et al. 1960). Aceordingly, rectilinear contours were eliminated by presenting the stimuli through a large translucent plastic tube, $1 \mathrm{~m}$ fong with a diameter of $61 \mathrm{~cm}$. Its far end subtended a circular 
a) 36 cke. The inside of the tube was lined with white paper to provide at contourless surface.

The subject sat in a high-backed wooden chair especially cumstructed $\mathbf{f}$ maintain him in a constant position during body tilt. li was provided with arm rests, a head restraint, and a chin clamp. The chair was atlached to the wall with a pirot directly behind the subject's head so that it could be rotated up to $45 \mathrm{deg}$ in both directions without lateral displacement of his head.

A complete response protile of the subjects's reports of fragmentations was obtained by having him signal partial or complete disappearance of the line stimulus by depressing a hand-held momentary contact switch for the duration of each fragnentation. It activated an event marker on a Grass 79.2 portable poligraph. This method of recording both partial and whole fades was adopted to replicate that used by Craig and Lichtemstein (1953). In fact, under the conditions used. the subjects reported relative! few whole fades: during postexperimental debricting. four subjects reported noticing no whole fades and no subject estimated them to be more than $10 \%$ of all fades reported.

\section{Procedure}

The subject is dominant eye was determined by having him adjus his head so that he could view a point of a pencil about $70 \mathrm{~cm}$ in front of him through a $5-\mathrm{mm}$ hole in a piece of paper held about halfin aty between him and the pencil. The alignment was made with both eyes upen. After alignnent, he was asked successively to close each eve alone. His dominant eye was taken to be the one for which closure blocked view of the point. The general experimental procedure was explained infornally to the subject at the beginning of the tirst experimental condition in the manner of Craig and Lichtenstein (1953). After he was seated in the chair and his head locked into a tixed position. he was given a chance to make several practice fixations. The stimulus was presented in two body-position conditions in counterbalanced order: body upright and boy tilted right-laterally 45 deg relative to gravity. In each condition, the stimulus. which was centered in front of the viewing eye, was presented randomly in nine different orientations relative to gravity from 0 to 180 deg in 22.5-deg steps. It was tixated for $50 \mathrm{sec}$ in each orientation. and fragmentations were reported. Each fixation period was alternated with 10 -sec rest periods and was presented in three blocks of nine trials for each condition. The overall order within each block was approximately counterbalanced across subjects. Since 45-sec time-outs were allowed between each block of tixations. each experimental session lasted approximately $35 \mathrm{~min}$. including the time to position the subject.

\section{RESULTS}

The propensity of the stimulus to fragment was assessed as a function of orientation in terms of three dependent variables: the average number of fragmentations per tixation period, the total duration of perceived fragmentation per fixation period, and the average latency to the first fragmentation after the onset of tixation. As Table 1 indicates, the three variables were highly intercorrelated for all subjects. A mixed-model analysis of variance was carried out for each of these variables using a four-way repeated-measures design. The nine grativational orientations of the stimulus constituted the levels of the stimulus position factor. The three time blocks (nine tixations each) constituted the three levels of the time factor. The body position factor levels were the two conditions used to view the stimulus. Since the order of the conditions was counterbalanced across
Table 1

\begin{tabular}{rccc} 
Subjects & $\begin{array}{c}\text { Number of Fragmentations } \\
\text { by } \\
\text { Duration of } \\
\text { Fragmentation }\end{array}$ & $\begin{array}{c}\text { Latency of } \\
\text { Fragmentation }\end{array}$ & $\begin{array}{c}\text { Duration of } \\
\text { Fragmentation } \\
\text { by } \\
\text { Latency of } \\
\text { Fragmentation }\end{array}$ \\
\hline 1 & .651 & -.435 & -.416 \\
2 & .766 & -3.85 & -.406 \\
3 & .517 & -.544 & $-.234 \mathrm{NS}$ \\
4 & .942 & -.642 & -.676 \\
5 & .830 & -.472 & -.465 \\
6 & .824 & -.543 & -.640 \\
7 & .819 & -.553 & -.606 \\
8 & .735 & -.438 & -.530 \\
9 & .844 & -.605 & -.709 \\
10 & .814 & -.398 & -.513 \\
11 & .552 & $-.313 \mathrm{~ns}$ & $-.316 \mathrm{~ns}$ \\
12 & .827 & -.601 & -.597 \\
13 & .893 & -.660 & -.792 \\
14 & .782 & -.670 & -.695 \\
\hline
\end{tabular}

Note-The intercorrelations between the three dependent variables used in the experiment are tabulated for all subjects. All correlations not marked by ns or NS are significant with a two-tailed test for $d f=52, p<.01: n s=.01<p<.05$, $N S=$ not significant.

subjects, a fourth factor could be assessed: order of body position factor. Subjects, who otherwise were crossed with all factors, were nested within this one in groups of seven.

Since the data were gathered in a repeatedmeasures design, it is prudent to test the $F$ ratios involving factors crossed with subjects using corrected degrees of freedom (Winer, 1971). This procedure will protect against spuriously significant $\mathrm{F}$ ratios due to heterogeneity of covariance. On all the dependent variables, the only main effect or interaction that remained signiticant after this conservative correction was the Stimulus Position by Body Position interaction (number of fragmentations: $F=5.53, \mathrm{df}$ $=8.96, \mathrm{p}<.001$, corrected $\mathrm{df}=1.12, \mathrm{p}<.04$; duration of fragmentation: $F=9.29$, df $=8,96$, $\mathrm{p}<.001$. corrected $\mathrm{df}=1.12, \mathrm{p}<.025$; latency to fragmentation: $F=3.61$, $\mathrm{df}=8.96, \mathrm{p}<.01$, corrected $\mathrm{df}=1.12$, n.s.). This interaction is displayed in Figure 1 without the latency data, since this dependent variable did not reach significance with the corrected degrees of freedom.

The data plotted in this figure clearly demonstrate the greater propensity of obliquely oriented line stimuli to fragment when fixated. Furthermore, this anisotropy of fragmentation is apparently determined by the retinal orientation of the stimuli. Had the gravitational orientation of the stimuli, indicated as stimulus position in the figure, been the determining factor, the plotted interaction should not occur. The curves for the different viewing conditions should have been coincident, and the only significant effect should have been that of stimulus position.

A retinal determination hypothesis in contrast predicts that the body tilt and upright curves should 


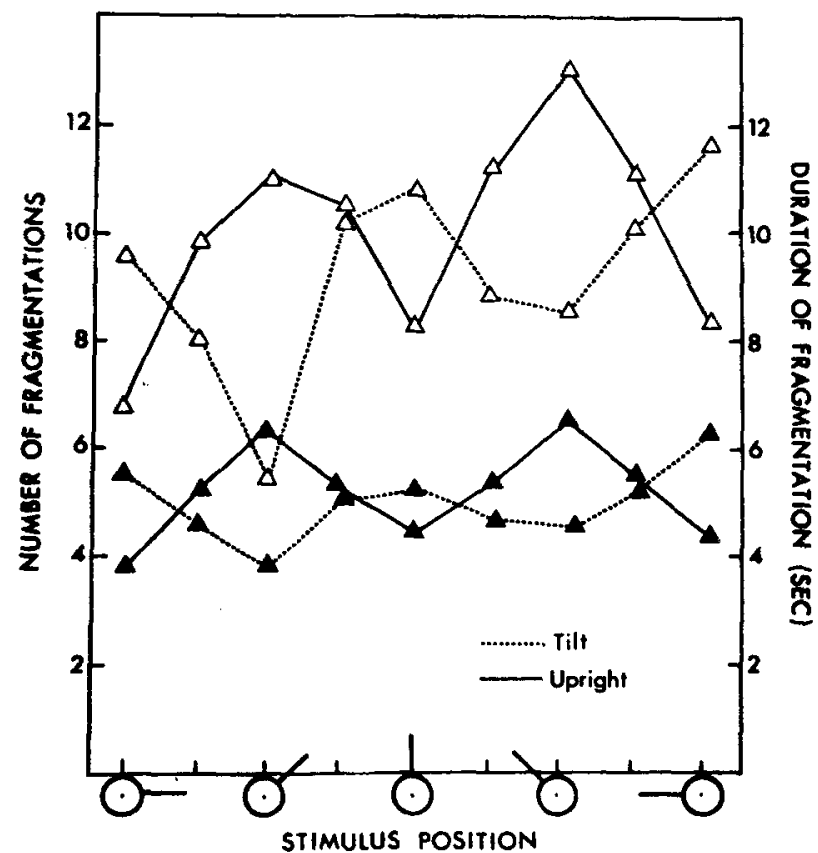

Figure 1. The acrous-mubject average propensity of fragmentation during the 50 -sec firation periods is plotted as a function for two dependent variables: the number of fragmentations per fixation period (filled symbols) and the total duration (seconds) of fragmentation per fixation period (open symbols). The dotted lines repreant the 45-deg right-lateral body-tilt condition; the solid lines represent the body-upright condition. The gravitational stimulus position bs schematically represented along the bottom of the graph.

be shifted with respect to each other exactly by the amount of retinal tilt produced by the body tilt. In this experiment, the retinal tilt would have been approximately $39.5 \mathrm{deg}$ due to the $5.5-\mathrm{deg}$ countertorsion that occurs during 45-deg lateral body tilts (Miller, 1962). Clearly, the results are most consistent with this retinal hypothesis. Nevertheless, the retinal orientation may not completely determine the results, since the effects of a small gravitational component may be masked by the sampling interval of $22.5 \mathrm{deg}$.

The following considerations provided evidence that this gravitational component must be small. The component would manifest itself as a partial orientation constancy of the "oblique effect." Consider the effects of such a partial constancy on the curvilinearity of the body-tilt curves in Figure 1. If, for example, a physical rotation of the retina of $45 \mathrm{deg}$ causes an opposite retinal rotation of the axes of minimum and maximum propensity for fragmentation of $22.5 \mathrm{deg}$, then the curvilinearity of these curves would be abolished: each gravitational orientation to which the subject was exposed would now fall exactly halfway between the axes of minimum and maximum propensity to fragment. Accordingly, it can be seen that the curvilinearity of the body-tilt curves would be a decreasing function of the retinal counterrotation of the axes of minium and maximum propensity to fragment in the range of counterrotations of 0 to $22.5 \mathrm{deg}$. Assuming that the propensity to fragment is inversely proportional to the visual acuity, an adaptation of Mansfield's (1974) anisotropy index can be used as a measure of curvilinearity. It conveniently describes the differences between visual function along the vertical/horizontal and oblique meridia. When used to compare the curvilinearity of body tilt and body upright curves for the duration of fragmentation variable, the adapted index yields a value of .49 for both curves. This is a bit surprising since the 5.5-deg countertorsion during body tilt could be expected to reduce the curvilinearity of the body tilt curve slightly. Assuming, for example, the reduction to be linearly proportional to the rotation for small rotations, the countertorsion would lead one to expect an index of .42. The number of fragmentations variable, in fact, shows an index reduction of this sort: the index value of .49 for the upright condition is reduced to .34 for the body-tilt condition. This reduction corresponds to a rotation of about $6.4 \mathrm{deg}$, 5.5 of which could be accounted for by countertorsion. Thus, the results of this experiment do not provide consistent evidence that the gravitational orientation of the stimulus is a major determining factor for the "oblique effect" observed by Craig and Lichtenstein (1953), though small effects on the order of 2-3 deg cannot be ruled out.

\section{DISCUSSION}

The fact that the propensity to fragment was measured mainly in terms of fragmentations, i.e., partial fades of the line stimulus, may be significant. Whole stimulus disappearances have been contrasted to partial disappearances as being differentially affected by patterned stimulation (Cosgrove. Schmidt, Fulgram, \& Brown, 1972). However, the differences reported are demonstrated during protracted stabilization using an optical-lever contact lens system. Accordingly, some of the differences could be attributed to the differential effects of lens slippage (Riggs \& Schick, 1968). In any case, the differences reported are not noted during the first few minutes of viewing, the period most relevant for comparison with the present experiment. In fact, during this initial viewing period, the measures of both partial and whole fades are highly correlated (also Schmidt, Fulgram, \& Brown, 1971). Nevertheless, reports indicate that adaptation to gratings before viewing stabilized lines only alters consistently the whole-fade frequency in an orientation-specific manner (Schmidt, Cosgrove, \& Brown, 1972). Thus, the possibility remains that under some conditions, whole and partial fades reflect different neural processes. However, since both types 
of fades exhibit similar variation as a function of retinal stimulus orientation (Schmidt, Fulgram, \& Brown. 1971). there is at least a prima facie case for both types retlecting the same visual anisotropy during a period of tixation less than $1 \mathrm{~min}$.

Another consideration regarding the results of the present experiment concerns the behavior of the subject's perception of the gravitational vertical during the protracted tilt. If the orientation of the "perceived" gravitational vertical changed during the tilt so as to become parallel with the retinal midline, the above results could be interpreted as indicating that the orientation of the stimulus relative to the "perceived" gravitational vertical was the factor determining rate of fragmentation. However, this major type of perceptual confusion has been shown not to occur under conditions of body tilt and degraded visual stimulation comparable to those used in this experiment (Wade, 1970).

The results of this study place the differential propensity of tixated line stimuli to fragment in the class of retinally locked "oblique effects." As such, it is possibly related to Manstield's (1974) report of anisotropy in the distribution of toveal receptive field orientation and may be considered primarily a visual phenomenon. Indeed, the present results as well as Cratig and Lichtenstein's (1953) cannot completely rule out the possibility that the anisotropy in fragmentation was caused by small, probably less than .25 diopter. uncorrected astigmatism either along the vertical or along the horizontal meridia. In view of the similarity between the observed fragmentation and Troxler's effect, it is tempting to view it as an example of this classic effect. However, the conventional location of Troxler's phenomenon in peripheral vision contrasts with the foveal/parafoveal location of stimuli exhibiting retinally locked "oblique effects."

The retinally locked feature of the observed differential fragmentation unequivocally differentiate it from other "oblique effects" such as the orientation-specitic variation in response latency reported by Attneave and Olson (1967). This latter effect is clearly determined by the perceptual rather than the retinal orientation of the stimuli. In contrast to the differential propensity to fragment, effects such as this probably reflect aspects of spatial perception mather than vision per se. For example, the longer reaction times that are reported associated with oblique stimuli may result from the mirror-image symmetry of the left and right facing obliques used. Such symmetry has been argued to increase stimulus contusability and to require special perceptual processing not needed for the recognition of vertical or horizontal stimuli (Corballis \& Beale, 1970; Howard \& Templeton, 1906).

In summary. the contrast between the differential fragmentation studies in this experiment and orientation-specific variation in response latency underlies a difference between different classes of "oblique effects," indicating the multiply determined character of this phenomenon. Furthermore, it indicates that at least one aspect of fragmentation phenomena nay be simply explained without reference to high-order, orientation-independent aspects of pattern perception.

\section{REFERENCES}

Appalle, S. Perception and discrimination as a function of stinulus orientation: The "oblique effect" in man and animals. Psychological Bulletin, 1972, 78, 266-278.

Attneave, F., \& Olson, R. Discriminability of stimuli varying in physical and retinal orientation. Journal of Experimental Psychology. 1967. 74. 149-157.

Attneave, F.. \& Reid. K. W. Voluntary control of frame of reference and slope equivalence under head rotation. Journal of Experimental Psychology, 1968, 78, 153-159.

Campbell, F. W., Kulikowski, J. J.. \& Levinson, J, The effect of orientation on the visual resolution of gratings. Journal of Physiology, 1966. 187. 427-436.

Campbell, F. W.. \& Maffei, L. Electrophysiological evidence for the existence of orientation and size detectors in the human lisual system. Joumal of Physiology, 1970, 207, 635-652.

Coltheart, M., \& CoOper, C. M. The retinal reference of the tilt aftereffect. Perception \& Psychophysics, 1972, 11, 312-324.

Corballis, M. C., \& Beale, I. L. Bilateral symmetry and behavior. Psychological Revien, 1970, 77, 451-464.

Cosgrove, M. P., Schmidt, M. J., Fulgram, D. D., \& Brown, R. Stabilized images: Dependent variable specificity of pattern effects with prolonged viewing. Perception \& Psychophysics, 1972, 11, 398-402.

Craig, E. A.. \& Lichtenstein, M. Visibility-invisibility cycles as a function of stimulus-orientation. American Journal of Psychology. 1953, 66, 554-563.

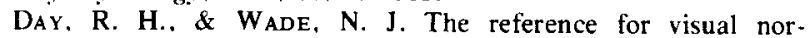
malization. American Joumal of Psychology, 1969, 82, 191-197.

DENNEY. D.. \& ADORJANI, C. Orientation speciticity of visual cortical neurons after head tilt. Experimental Brain Research, 1972, 14. 312-317.

Donderi. D. C., \& Kane. E. Perceptual learning produced by common responses to different stimuli. Canadian Journal of Psychology. 1965. 19. 15-40.

Ellis. S. R. Investigations of the orientation-constancy of two visual aftereffects. Doctoral dissertation. McGill University. 1974.

Ferree, C. E., Rand. G.. \& Hardy. C. An important factor in space perception in the peripheral field of vision. American Journal of Psichology, 1933, 45, 228-247.

Findlay. J. M.. \& Parker. D. M. An investigation of visual orientation constancy using orientation-specitic properties of acuity and adaptation. Perception. 1972. 1. 305-313.

Gibson. J. J. Adaptation with negative after-effect. Psychological Review. 1937. 44. 222-244.

Gibson. J. J.. \& Radner, M. Adaptation, after-effect, and contrast in the perception of tilted lines. I. Quantitative studies. Jom mal of Experime'ntal Psichology. 1937, 20. 453-467.

HEBB. D. O. The semiautononous process: Its nature and nurture. American Psychologist, 1963, 18, 16-26.

Higgins. G. C.. \& Stultz, K. Visual acuity as measured with various orientations of a parallel-line test object. Joumal of the Oprical Society of America. 1948, 38, 756-758.

Howard, I. P.. \& Templeton. W. B. Human spatial orientution. Now York: Wiley, 1966. 
Horn, G., \& Hill, R. M. Modifications of receptive fields of cells in the visual cortex occurring spontaneously and associated with body tilt. Nature, 1969. 221, 186-188.

Horn, G. Stechler, G., \& Hile, R. M. Receptive fields of units in the visual cortex of the cat in the presence and absence of bodily tilt. Experimental Brain Research, 1972, 15, 113-132.

Jung, R., Kornhuber, H. H., \& de Fonseca, J. S. Multisensory convergence on cortical neurons. Progress in Brain Research and Brain Mechanisms, 1\%3, 1, 207-234.

LENNIE, P. Head orientation and meridional variations in acuity. Vision Research, 1974, 14, 107-111.

LURIA, S. M. The effect of body position on meridional variations in scotopic acuity, American Journal of Psychology, $1963,76,598-406$.

Maffei, L., \& Campbelt, F. W. Neurophysiological localization of vertical and horizontal visual coordinates in man. Science, $1970,167,386-387$.

MANSfield, R. J. W. Neural basis of orientation perception in primate vision. Science, 1974, 186, 1133-1135.

Miller, E. F., l1. Counterrolling of the human eyes produced by head tilt with respect to gravity. Acta Oto-laryngology, $1962,54,479-501$.

Millodot, M., \& Lamont, A. Refraction of the periphery of the eye. Joumal of the Optical Society of America, 1974, 64, 110-111.

Mitchell, D. E. \& Blakemore, C. The site of orientation constancy. Perception, 1972, 1. 315-320.

Mitchell, D. E., Freeman, R. D., \& Westheimer, G. Effect of orientation on the modulation sensitivity for interference fringes on the retina. Journal of the Optical Society of America. 1967. 57. 246-249.

Ogilvie, J. C., \& Tarlor, M. M. Effect of orientation on the visibility of fine wires. Journal of the Optical Society of America, 1958, 48, 628-629.

Prentice, W. C. H., \& Bearoslee, D. C. Visual "normalization" near the vertical and horizontal. Joumal of Experimental Psychology, 1950, 40, 355-364.

Prichard, R., Heron, W., \& Hebs, D. O. Visual perception approached by the method of stabilized images. Canadian Journal of Psychology, 1960, 14, 67-77.

Riggs. L. A.. \& Schick, A. M. L. Accuracy of retinal image stabilization achieved with a place mirror in a tightly fitting contact lens. Vision Research, 1968, 8, 159-169.

Schmidt, M. J., Cosgrove, M. P., \& Brown, D. R. Stabilized images: Functional relationships among populations of orientation-specific mechanisms in the human visual system. Perception \& Psychophysics, 1972, 11, 389-392.
Schmidt, M. J.. Fulgram, D. D., \& Brown. D. R. Stabilized images: The search for pattern elements. Perception \& Psychophysics, 1971. 10. 295-299.

Schwartzkroin, P. A. The effect of body tilt on the directionality of units in the cat visual cortex. Experimental Neurology, 1972, 36, 598-606.

Silny, F. G., \& Kushnir, S. L. Effects of lateral body tilt on visibility-invisibility cycles as a function of stimulus orientation. Undergraduate honors thesis, Psychology Department. McGill University, 1971.

TAYLOR. M. M. Visual discrimination and orientation. Journal of the Optical Society of America, 1963, 53, 763-765.

TroxLER, D. Über das Verschwinden gegebener Gegenstände innerhalb unsers Gesichtskreises. Ophthalmologisches Bibliothek (Himly and Schmidt. Eds.. Jena), 1804, 2. 51-53.

WADE, N. J. Effect of prolonged tilt on visual orientation. Quarterly Journal of Experimental Psychology. 1970, 22, 423-439.

WINER, B. J. Statistical principles in Experimental design (2nd ed.). New York: McGraw-Hill. 1971.

\section{NOTES}

1. A notable difference between Luria's experiment and others is that he used scotopic conditions: it is, however, difficult to see why such a difference should be important.

2. The present experinent is an outgrowth of a similar experiment suggested by the author as an undergraduate honors thesis to two MeGill University undergraduates: Fredrick Silny and Seynour L. Kushnir (1971). Their experimental results, however, could not be unambiguously interpreted. as their failure to use a chin clamp may have allowed the subject's head to counterrotate during the body tilt.

3. Contrast $=[$ luminance $($ maximum $)-$ luminance $($ minimum $)]$ [luminance(maximum) + luminance(minimum)].

4. Anisotropy index $=1-\left\{\right.$ [visual acuity $\left(0^{\circ}\right)+$ visual acuity $\left.\left(90^{\circ}\right)\right] /\left[\right.$ visual acuity $\left(45^{\circ}\right)+$ visual acuity $\left.\left.\left(135^{\circ}\right)\right]\right\}$; for the adapted anisotropy index, let visual acuity $\left(X^{\circ}\right)=[1] /$ [fragmentation measure $\left(\mathrm{X}^{\circ}\right)$ ].

(Received for publication January 31, 1975; revision accepted April 17, 1975.) 